\title{
Differential and Functional Identities for the Elliptic Trilogarithm ${ }^{\star}$
}

\author{
Ian A.B. STRACHAN
}

Department of Mathematics, University of Glasgow, Glasgow G12 8QQ, UK

E-mail: i.strachan@maths.gla.ac.uk

URL: http://www.maths.gla.ac.uk/ iabs/

Received November 25, 2008, in final form March 06, 2009; Published online March 13, 2009

doi:10.3842/SIGMA.2009.031

\begin{abstract}
When written in terms of $\vartheta$-functions, the classical Frobenius-Stickelberger pseudo-addition formula takes a very simple form. Generalizations of this functional identity are studied, where the functions involved are derivatives (including derivatives with respect to the modular parameter) of the elliptic trilogarithm function introduced by Beilinson and Levin. A differential identity satisfied by this function is also derived. These generalized Frobenius-Stickelberger identities play a fundamental role in the development of elliptic solutions of the Witten-Dijkgraaf-Verlinde-Verlinde equations of associativity, with the simplest case reducing to the above mentioned differential identity.
\end{abstract}

Key words: Frobenius manifolds; WDVV equations; Jacobi groups; orbit spaces

2000 Mathematics Subject Classification: 11F55; 53B50; 53D45

\section{Introduction}

Amongst the many beautiful identities satisfied by the elliptic (and related) functions is the Frobenius-Stickelberger relation [12, 22]

$$
(\zeta(a)+\zeta(b)+\zeta(c))^{2}=\wp(a)+\wp(b)+\wp(c), \quad(a+b+c=0) .
$$

Writing this identity not in terms of Weierstrass functions but in terms of $\vartheta$-functions yields the equivalent form

$$
\left[\frac{\vartheta_{1}^{\prime}(a)}{\vartheta_{1}(a)} \frac{\vartheta_{1}^{\prime}(b)}{\vartheta_{1}(b)}+\frac{\vartheta_{1}^{\prime}(b)}{\vartheta_{1}(b)} \frac{\vartheta_{1}^{\prime}(c)}{\vartheta_{1}(c)}+\frac{\vartheta_{1}^{\prime}(c)}{\vartheta_{1}(c)} \frac{\vartheta_{1}^{\prime}(a)}{\vartheta_{1}(a)}\right]+\frac{1}{2}\left[\frac{\vartheta_{1}^{\prime \prime}(a)}{\vartheta_{1}(a)}+\frac{\vartheta_{1}^{\prime \prime}(b)}{\vartheta_{1}(b)}+\frac{\vartheta_{1}^{\prime \prime}(c)}{\vartheta_{1}(c)}\right]=\frac{1}{2} \frac{\vartheta_{1}^{\prime \prime \prime}(0)}{\vartheta_{1}^{\prime}(0)},
$$

where again $a+b+c=0$. With the help of the heat equation the second set of terms may be written in terms of derivatives with respect to the modular parameter $\tau$.

The purpose of this paper is to explore certain neo-classical identities satisfied by the elliptic trilogarithm introduced by Beilinson and Levin $[1,14]$. This function will be defined in Section 2, but for now it is sufficient to note that with it the above identity takes the simplified form

$$
\left\{\begin{array}{r}
f^{(3,0)}(a) f^{(3,0)}(b) \\
+f^{(3,0)}(b) f^{(3,0)}(c) \\
+f^{(3,0)}(c) f^{(3,0)}(a)
\end{array}\right\}-\left\{f^{(2,1)}(a)+f^{(2,1)}(b)+f^{(2,1)}(c)\right\}=0 .
$$

These new identities take the schematic form

$$
\{\text { quadratic terms }\}+\{\text { linear terms }\}=\{\vartheta \text { constants }\}
$$

* This paper is a contribution to the Proceedings of the Workshop "Elliptic Integrable Systems, Isomonodromy Problems, and Hypergeometric Functions" (July 21-25, 2008, MPIM, Bonn, Germany). The full collection is available at http://www.emis.de/journals/SIGMA/Elliptic-Integrable-Systems.html 
where the linear terms contain one more $\tau$-derivative than the total number of $\tau$-derivatives in each part in the quadratic term. Before these identities are discussed (Section 4) a differential equation satisfied by this function $f(z, \tau)$ will be derived in Section 3. Applications of these new identities are then discussed in Section 5 . We begin by defining the elliptic polylogarithm.

\section{The elliptic polylogarithms}

The classical polylogarithm is defined, for $|z|<1$, by

$$
\operatorname{Li}_{r}(z)=\sum_{n=1}^{\infty} \frac{z^{n}}{n^{r}}
$$

and by analytic continuation elsewhere. A first attempt at an elliptic analogue of this function might be

$$
\mathcal{L} \mathrm{i}_{r}(\zeta, q)=\sum_{n=-\infty}^{\infty} \operatorname{Li}_{r}\left(q^{n} \zeta\right)
$$

However this series diverges, but by using the inversion formula (11) and $\zeta$-function regularization one can arrive at the following definition of the elliptic polylogarithm function $[1,14]$ :

$$
\mathcal{L} \mathrm{i}_{r}(\zeta, q)=\sum_{n=0}^{\infty} \operatorname{Li}_{r}\left(q^{n} \zeta\right)+\sum_{n=1}^{\infty} \operatorname{Li}_{r}\left(q^{n} \zeta^{-1}\right)-\chi_{r}(\zeta, q), \quad r \text { odd }
$$

where

$$
\chi_{r}(\zeta, q)=\sum_{j=0}^{r} \frac{B_{j+1}}{(r-j) !(j+1) !}(\log \zeta)^{(r-j)}(\log q)^{j} .
$$

A real-valued version of this function had previously been studied by Zagier [23]. With this the function $f$ may be defined.

Definition 1. The function $f(z, \tau)$, where $z \in \mathbb{C}, \tau \in \mathbb{H}$, is defined to be:

$$
f(z, \tau)=\frac{1}{(2 \pi i)^{3}}\left\{\mathcal{L} \mathrm{i}_{3}\left(e^{2 \pi i z}, q\right)-\mathcal{L} \mathrm{i}_{3}(1, q)\right\} .
$$

The function $f^{(n, m)}$ that appear in the introduction are the derivatives

$$
f^{(n, m)}=\frac{\partial^{n+m} f}{\partial z^{n} \partial \tau^{m}}
$$

It immediately follows from the definition that

$$
\left(\frac{d}{d \tau}\right)^{3} \frac{1}{(2 \pi i)^{3}} \mathcal{L} \mathrm{i}_{3}(1, q)=\frac{1}{120} E_{4}(\tau) .
$$

and

$$
\left(\frac{\partial}{\partial z}\right)^{2} f(z, \tau)=-\frac{1}{2 \pi i} \log \left\{\frac{\vartheta_{1}(z, \tau)}{\eta(\tau)}\right\} .
$$

Thus the elliptic-trilogarithm may be thought of as a classical function (or, at least, a neoclassical function) as it may be obtained from classical elliptic functions via nested integration and other 
standard procedures. It does, however, provide a systematic way to deal with the arbitrary functions that would appear this way.

The following proposition describes the fundamental transformation properties of the function: these will be used in subsequent section to prove the various differential and functional identities. The precise definitions and normalizations of the various objects used here are given in Appendix A. Also, the notation $F \simeq G$ will be used if the functions $F$ and $G$ differ by a quadratic function in the variables $z$ and $\tau$. These quadratic terms may be easily derived, but will play no part in the rest of the paper.

Proposition 1. The function $f$ has the following transformation properties:

$$
\begin{aligned}
& f(z+1, \tau) \simeq f(z, \tau) \\
& f(z, \tau+1)=f(z, \tau) \\
& f(z+\tau, \tau) \simeq f(z, \tau)+\left\{\frac{1}{6} z^{3}+\frac{1}{4} z^{2} \tau+\frac{1}{6} z \tau^{2}+\frac{1}{24} \tau^{3}\right\} \\
& f(-z, \tau) \simeq f(z, \tau) .
\end{aligned}
$$

The function also has the alternative expansions:

$$
f(z, \tau) \simeq-\frac{1}{(2 \pi i)}\left\{\frac{1}{2} z^{2} \log z+z^{2} \log \eta(\tau)\right\}+\frac{1}{(2 \pi i)^{3}} \sum_{n=1}^{\infty} \frac{(-1)^{n} E_{2 n}(\tau) B_{2 n}}{(2 n+2) !(2 n)}(2 \pi z)^{2 n+2}
$$

and

$$
f(z, \tau)=\frac{1}{(2 \pi i)^{3}} \operatorname{Li}_{3}\left(e^{2 \pi i z}\right)+\frac{1}{12} z^{3}-\frac{1}{24} z^{2} \tau-\frac{4}{(2 \pi i)^{3}} \sum_{r=1}^{\infty}\left\{\frac{q^{r}}{\left(1-q^{r}\right)}\right\} \frac{\sin ^{2}(\pi r z)}{r^{3}} .
$$

The first of these imply the following the transformation property:

$$
f\left(\frac{z}{\tau},-\frac{1}{\tau}\right) \simeq \frac{1}{\tau^{2}} f(z, \tau)-\frac{1}{\tau^{3}} \frac{z^{4}}{4 !} .
$$

Proof. The first three relation follow immediately from the definition. The fourth used the inversion formula for polylogarithms (11).

The proof of (5) and (6) just involves some careful resumming. Consider the first two terms in the definition of $f$ :

$$
\sum_{n=0}^{\infty} \operatorname{Li}_{3}\left(q^{n} e^{2 \pi i z}\right)+\sum_{n=1}^{\infty} \operatorname{Li}_{3}\left(q^{n} e^{-2 \pi i z}\right)=\operatorname{Li}_{3}\left(e^{2 \pi i z}\right)+2 \sum_{s=0}^{\infty} \frac{(-1)^{s}}{(2 s) !}\left\{\sum_{n, r=1}^{\infty} q^{n r} r^{2 s-3}\right\}(2 \pi z)^{2 s} .
$$

From this series (6) follows immediately. To obtain (5) one rearranges the terms. The $s=0$ term cancels in the final expression and the remaining terms may be re-expressed in terms of Eisenstein series (for $s>1$ ) or the Dedekind function (for $s=1$ ). Finally, using the result

$$
\frac{1}{(2 \pi i)^{3}} \frac{d^{3}}{d z^{3}} \operatorname{Li}_{3}\left(e^{2 \pi i z}\right)=-\frac{1}{2}[1+\operatorname{coth}(\pi i z)]=-\left[\frac{1}{2}+\frac{1}{(2 \pi z)}+\sum_{n=1}^{\infty} \frac{B_{2 n}}{(2 k) !}(2 \pi i z)^{2 k-1}\right]
$$

one may obtain a series for $\operatorname{Li}_{3}\left(e^{2 \pi i z}\right)$. Putting all these parts together gives the series (5). 


\section{Differential identities}

Theorem 1. The function $f$ satisfies the equation

$$
f^{(3,0)} f^{(1,2)}-\left(f^{(2,1)}\right)^{2}+\frac{1}{3} f^{(0,3)}=-\frac{1}{144} E_{4}(\tau) .
$$

Proof. We denote the left hand side of the differential equation by $\Delta(f)(z, \tau)$ and study its transformation properties. It follows from Proposition 1 that the third derivatives of $f$ are invariant under the transformation $z \mapsto z+1$ and that under the transformation $z \mapsto z+\tau$ one has:

$$
\begin{aligned}
& f^{(3,0)}(z+\tau)=f^{(3,0)}(z)+1 \\
& f^{(2,1)}(z+\tau)=f^{(2,1)}(z)-f^{(3,0)}(z)-\frac{1}{2} \\
& f^{(1,2)}(z+\tau)=f^{(1,2)}(z)-2 f^{(2,1)}(z)+f^{(3,0)}(z)+\frac{1}{3}, \\
& f^{(0,3)}(z+\tau)=f^{(0,3)}(z)-3 f^{(1,2)}(z)+3 f^{(2,1)}(z)-f^{(3,0)}(z)-\frac{1}{4} .
\end{aligned}
$$

It immediately follows that combination $\Delta(f)$ is a doubly periodic function (even though individual components are not). From the Laurent expansions of $f$, the only term with a pole is $f^{(3,0)}$, which has a simple pole at $z=0$, but this cancels with the zero at $z=0$ of the term $f^{(1,2)}$. Thus $\Delta(f)$ is a doubly period function with no poles, and hence must be independent of $z$, i.e. a $\vartheta$-constant.

Using the series expansion in Proposition 1 one finds that

$$
\Delta=\frac{1}{12}\left\{\frac{1}{2 \pi i} E_{2}^{\prime}-\frac{1}{12} E_{2}^{2}\right\}
$$

and hence the result follows on using the Ramanujan identity

$$
q \frac{d E_{2}}{d q}=\frac{E_{2}^{2}-E_{4}}{12}
$$

An alternative proof is to study the modularity properties of $\Delta$ under $\tau \rightarrow-\tau^{-1}$. One finds that $\Delta$ must be a modular form of degree 4 , and hence must be a multiple of $E_{4}$, the constant of proportionality being straightforward to calculated. Note, one could easily redefine $f$, using (4), so that $\Delta=0$.

This differential identity contains much information. Using the $z$-expansion of the function $f$ it is equivalent to an infinite family of identities between the Eisenstein series and their derivatives, though these will all be consequences of the Ramanujan relations and the fact that the ring of modular forms is finitely generated by $E_{4}$ and $E_{6}$. Alternatively, using the $q$-expansion of $f$ it is equivalent to an infinite family of number-theoretic trigonometric identities (number theoretic since in the $q$-series for $f$ the $q^{n}$ coefficient will involve a sum over $\left.r \mid n\right)$.

\section{Functional identities and rank 2-root systems}

The basic identity (2) may be associated to the $A_{2}$-root system via the identification $a=(\alpha, \mathbf{z})$, $b=(\beta, \mathbf{z}), c=-(\alpha+\beta, \mathbf{z})$, where $\alpha$ and $\beta$ are the positive roots and $(\cdot, \cdot)$ is the standard Euclidean inner product. This immediately suggest that there should be variants of this identity for other rank-2 root systems. 
Lemma 1. Let $\mathcal{R}$ be the root system for the 2-dimensional Coxeter groups $A_{2}, B_{2}$ or $G_{2}$, with the standard normalization for $\alpha, \beta$ positive simple roots:

$$
\begin{array}{lll}
A_{2}: & (\alpha, \alpha)=(\beta, \beta)=2, \quad(\alpha, \beta)=-1, \\
B_{2}: & (\alpha, \alpha)=2, \quad(\beta, \beta)=1, \quad(\alpha, \beta)=-1, \\
G_{2}: & (\alpha, \alpha)=6, \quad(\beta, \beta)=2, \quad(\alpha, \beta)=-3 .
\end{array}
$$

Then

$$
\sum_{\alpha \neq \beta \in \mathcal{R}^{+}}(\alpha, \beta) f^{(3,0)}((\mathbf{z}, \alpha), \tau) \cdot f^{(3,0)}((\mathbf{z}, \beta), \tau)+\sum_{\alpha \in \mathcal{R}^{+}} k_{\alpha} f^{(2,1)}((\mathbf{z}, \alpha), \tau)=0,
$$

where:

- $A_{2}: k_{\alpha}=1$ for all roots;

- $B_{2}: k_{\text {short }}=2, \quad k_{\text {long }}=1$;

- $G_{2}: k_{\text {short }}=10, \quad k_{\text {long }}=6$.

The proof is entirely standard and is omitted: it follows the proof of Theorem 1; one first shows that the left hand side is doubly periodic in all $a, b, c$ variables (constrained via $a+b+c=0$ ) with no poles in all these variables, and hence must be independent of all three variables, i.e. it must be a $\vartheta$-constant. Modular properties then fix this $\vartheta$-constant.

Many other functional identities may be derived using the same ideas. We present here a (not exhaustive) list of identities, all of the general form (3), for the root system $A_{2}$ and $B_{2}$ (see Appendix B $)^{1}$. The origin of such identities stems from the analysis of elliptic solutions to the Witten-Dijkgraaf-Verlinde-Verlinde equation. This application will be presented in the next section.

\section{1 $\quad A_{2}$-identities}

$$
\begin{aligned}
& \left\{\begin{array}{c}
f^{(3,0)}(x+y)\left[f^{(2,1)}(x)-f^{(2,1)}(y)\right] \\
+f^{(3,0)}(y)\left[f^{(2,1)}(x+y)-f^{(2,1)}(x)\right]
\end{array}\right\} \\
& +\left\{f^{(1,2)}(x)-\frac{1}{2} f^{(1,2)}(y)+\frac{1}{2} f^{(1,2)}(x+y)\right\}=0, \\
& \left\{\begin{array}{c}
f^{(3,0)}(x)\left[f^{(1,2)}(x+y)-f^{(1,2)}(y)\right] \\
+f^{(3,0)}(y)\left[f^{(1,2)}(x+y)-f^{(1,2)}(x)\right] \\
-\frac{2}{3} f^{(3,0)}(x+y)\left[f^{(1,2)}(x)+f^{(1,2)}(y)\right]
\end{array}\right\}+\left\{\begin{array}{c}
\frac{2}{3} f^{(2,1)}(x+y) f^{(2,1)}(x) \\
+\frac{2}{3} f^{(2,1)}(x+y) f^{(2,1)}(y) \\
-\frac{8}{3} f^{(2,1)}(x) f^{(2,1)}(y)
\end{array}\right\} \\
& +\frac{10}{9} f^{(0,3)}(x+y)+\frac{1}{108} E_{4}(\tau)=0 .
\end{aligned}
$$

The dependence of $f$ on $\tau$ in these formulae has been suppressed for notational convenience.

\section{Applications}

In this section we present two applications of these identities: one is in the theory of elliptic solutions to the Witten-Dijkgraaf-Verlinde-Verlinde (or WDVV) equations of topological quantum field theory [19], and the second, more speculative and incomplete, in the theory of elliptic Dunkl-type operators.

\footnotetext{
${ }^{1}$ The corresponding identities for $G_{2}$ are available on request.
} 


\subsection{Frobenius manifolds and solutions to the WDVV-equations}

The basic identity (2) has particularly simple rational and trigonometric limits. Given $a, b, c \in \mathbb{C}$ such that $a+b+c=0$ then the rational limit gives

$$
\frac{1}{a} \cdot \frac{1}{b}+\frac{1}{b} \cdot \frac{1}{c}+\frac{1}{c} \cdot \frac{1}{a}=0
$$

and the trigonometric limit gives

$$
\cot (a) \cot (b)+\cot (b) \cot (c)+\cot (c) \cot (a)=1 .
$$

These identities are well known, and one application of them is in the construction of solutions to the WDVV-equation. In particular, the function

$$
F=\frac{1}{4} \sum_{\alpha \in \mathcal{R}_{W}}(\alpha, \mathbf{z})^{2} \log (\alpha, \mathbf{z})^{2}
$$

satisfies the WDVV equation (see, for example, $[3,15]$ )

$$
\left[\mathbf{F}_{i}, \mathbf{F}_{j}\right]=\mathbf{0}
$$

where

$$
\left(\mathbf{F}_{i}\right)_{j}^{k}=g^{k r} \frac{\partial^{3} F}{\partial z^{i} \partial z^{j} \partial z^{r}},
$$

(here $g$ is the Euclidean metric) and $\mathcal{R}_{W}$ are the roots of a Coxeter group $W$. The verification of this result reduces to sums over vectors $\mathcal{R}_{W} \cap \mathfrak{U}$, where $\mathfrak{U}$ is an arbitrary 2-plane, and the only non-trivial configurations are the 3 irreducible 2 -dimensional root systems. The WDVV equations are then satisfied by virtue of identities such as (8). Similarly, the verification that the trigonometric function $[10,16]$

$$
F=\text { cubic terms }+\sum_{\alpha \in \mathcal{R}_{W}} h_{\alpha} \operatorname{Li}_{3}\left(e^{i(\alpha, \mathbf{x})}\right)
$$

satisfies the WDVV equation involves use of identities of the form (9). It is not therefore surprising that the elliptic identities of the form (2) should play a role in elliptic solutions of the WDVV equation. Indeed, the origin of the differential equation in Theorem 1 is the following:

Lemma 2. The function

$$
F(u, z, \tau)=\frac{1}{2} u^{2} \tau-u z^{2}+h(z, \tau)
$$

satisfies the WDVV equations if and only if $h(z, \tau)$ satisfies the partial differential equation

$$
h^{(3,0)} h^{(1,2)}-\left(h^{(2,1)}\right)^{2}+\frac{1}{3} h^{(0,3)}=0 .
$$

From Theorem 1, one solution of this equation is clearly

$$
h(z, \tau)=f(z, \tau)+\frac{5}{2(2 \pi i)^{3}} \mathcal{L} \mathrm{i}_{3}(1, q) .
$$

However, another solution is

$$
h(z, \tau)=f(2 z, \tau)-4 f(z, \tau)
$$


and it is this solution that corresponds to a nice geometric structure, namely the dual prepotential to the $A_{1}$-Jacobi group orbit space [17]. This, and the corresponding potentials that can be calculated for the $A_{N^{-}}$Jacobi group orbit [17] space suggest that one can develop the following functional ansatz for more general class of solutions:

$$
F(u, \mathbf{z}, \tau)=\frac{1}{2} u^{2} \tau-\frac{1}{2} u(\mathbf{z}, \mathbf{z})+\sum_{\alpha \in \mathfrak{U}} h_{\alpha} f((\mathbf{z}, \alpha), \tau) .
$$

Here $(u, \mathbf{z}, \tau) \in \mathbb{C} \times \mathbb{C}^{N} \times \mathbb{H}$ and $\mathfrak{U}$ is a certain finite set of 'root vectors' in $\mathbb{C}^{N}$. Note that the vector field $e=\frac{\partial}{\partial u}$ is the unity of the multiplication, and that

$$
g=2 d u d \tau-(d \mathbf{z}, d \mathbf{z}),
$$

where $($,$) is the standard complexified Euclidean inner product on \mathbb{C}^{N}$. The precise conditions on the set of vectors $\mathfrak{U}$ which are required for (10) to satisfy the WDVV equations were derived in [19]. These start with the definition of a complex Euclidean $\vee$-system:

Definition 2 ([11]). Let $\mathfrak{h}$ be a complex vector space with non-degenerate bilinear form $($, and let $\mathfrak{U}$ be a collection of vectors in $\mathfrak{h}$. A complex Euclidean $\vee$-system $\mathfrak{U}$ satisfies the following conditions:

- $\mathfrak{U}$ is well distributed, i.e. $\sum_{\alpha \in \mathfrak{U}} h_{\alpha}(\alpha, \mathbf{u})(\alpha, \mathbf{v})=2 h_{\mathfrak{U}}^{\vee}(\mathbf{u}, \mathbf{v})$ for some $\lambda$;

- on any 2-dimensional plane $\Pi$ the set $\Pi \cap \mathfrak{U}$ is either well distributed or reducible (i.e. the union of two non-empty orthogonal subsystems).

With this one can define an elliptic $\vee$-system as follows:

Definition 3. An elliptic $\vee$-system $\mathfrak{U}$ is a complex Euclidean $\vee$-system with the following additional conditions:

- $\sum_{\alpha \in \mathfrak{U}} h_{\alpha}(\alpha, \mathbf{z})^{4}=3(\mathbf{z}, \mathbf{z})^{2}$;

- the three conditions:

$$
\begin{aligned}
& \sum_{\beta \in \Pi_{\alpha} \cap \mathfrak{U}} h_{\beta}(\alpha, \beta)\left(\beta, \alpha^{\perp}\right)^{n}=0, \\
& \sum_{\beta \in \Pi_{\alpha} \cap \mathfrak{U}} h_{\beta}(\alpha, \beta)(\alpha \wedge \beta)\left(\beta, \alpha^{\perp}\right)^{n}=0, \\
& \sum_{\beta \in \Pi_{\alpha} \cap \mathfrak{U}} h_{\beta}(\alpha, \beta)(\alpha \wedge \beta) \otimes(\alpha \wedge \beta)\left(\beta, \alpha^{\perp}\right)^{n}=0 ;
\end{aligned}
$$

- there exists a full $N$-dimensional weight lattice of vectors $\mathbf{p}$ such that $(\mathbf{p}, \alpha) \in \mathbb{Z}$ for all $\alpha \in \mathfrak{U}$.

With this definition of an elliptic $\vee$-system one can state the main theorem of [19].

Theorem 2. Let $\mathfrak{U}$ be an elliptic $\vee$-system. If $h_{\mathfrak{U}}^{\vee}=0$ then the function

$$
F(u, \mathbf{z}, \tau)=\frac{1}{2} u^{2} \tau-\frac{1}{2} u(\mathbf{z}, \mathbf{z})+\sum_{\alpha \in \mathfrak{U}} h_{\alpha} f((\mathbf{z}, \alpha), \tau)
$$

satisfies the $W D V V$ equations. If $h_{\mathfrak{U}}^{\vee} \neq 0$ then the modified prepotential

$$
F \longrightarrow F+\frac{10\left(h_{\mathfrak{U}}^{\vee}\right)^{2}}{3(2 \pi i)^{3}} \mathcal{L i}_{3}(1, q)
$$

satisfies the WDVV equations. 
The proof of this theorem is entirely analogous to the proof of Theorem 1: the first and third parts of Definition 3 ensure that that functions $\boldsymbol{\Delta}=\left[\mathbf{F}_{i}, \mathbf{F}_{j}\right]$, the obstructions to the WDVVequations from holding, are doubly periodic in all $\mathbf{z}$-variables, and the second conditions ensure that these functions have no poles and hence must be a $\vartheta$-constant. Modular properties then fix the $\vartheta$-constant as above, and hence to the vanishing of the obstructions $\boldsymbol{\Delta}$.

One unexpected result is the a 'pure' root system does not suffice: it is not, in general, an elliptic $\vee$-system. Thus generalization of classical integrable systems from rational and trigonometric to elliptic based purely on Coxeter root systems may not always work.

\subsection{Dunkl-type operators}

There is a close similarity between the mathematics behind the WDVV equations and the commutativity of Dunkl operators. Given the role of the above functional identities in the construction of solutions to the associativity equations a natural question to raise is whether or not they have a role in Dunkl-type operators. The results presented here will be for the Coxeter group $A_{2}$ alone.

Let

$$
\begin{aligned}
& \Xi^{(-1)}(\xi)=\partial_{\xi}+\sum_{a \in \mathfrak{U}} k_{a}(a, \xi) \hat{s}_{a}, \\
& \Xi^{(i)}(\xi)=\partial_{\xi}+\sum_{a \in \mathfrak{U}} k_{a}(a, \xi) f^{(3-i, i)}(\mathbf{z}, a) \hat{s}_{a}, \quad i=0,1,2,3 .
\end{aligned}
$$

Here $\mathfrak{U}=\{\alpha, \beta,-(\alpha+\beta)\}$ where $\alpha$ and $\beta$ are the simple positive roots of $A_{2}$. The following follows from direct calculation, on using the basic identity (2).

Proposition 2. Let

$$
\mathbb{F}^{(a, b)}(\xi, \eta)=\left[\Xi^{(a)}(\xi), \Xi^{(b)}(\eta)\right]+\left[\Xi^{(b)}(\xi), \Xi^{(a)}(\eta)\right] .
$$

Then

$$
\begin{aligned}
& \mathbb{F}^{(0,-1)}(\xi, \eta)=0, \\
& \mathbb{F}^{(0,0)}(\xi, \eta)+\mathbb{F}^{(1,-1)}(\xi, \eta)=0 .
\end{aligned}
$$

(Note that the second of these can be written more succinctly as

$$
g_{\mu \nu}\left[\Xi^{(\mu)}(\xi), \Xi^{(\nu)}(\eta)\right]=0
$$

where $g=d u d \tau+2 d z^{2}$.) These relations bears a close similarity with the zero-curvature relations of the elliptic WDVV equations. However, terms such as $\mathbb{F}^{(0,1)}(\xi, \eta)$ cannot be expressed in terms of other $\mathbb{F}^{(i, j)}$, which might have been expected via the use of the generalized FrobeniusStickelberger relation (7).

These operators $\Xi$ do have simple rational and trigonometric limits, from which one can recover standard results. In the rational limit $f^{(3,0)}(z, \tau) \sim z^{-1}$ and one hence obtains the original result [5] of Dunkl

$$
\left[\Xi^{(0)}(\xi), \Xi^{(0)}(\eta)\right]=0
$$

and in the trigonometric limit $(q \rightarrow 0) f^{(2,1)}(z, \tau) \rightarrow-\frac{1}{12}$ and so

$$
\Xi^{(1)}(\xi) \rightarrow-\frac{1}{12} \Xi^{(-1)}(\xi)
$$


With this one obtains Heckman's operators [13] which, following Cherednik, can be made into a pair of commuting operators [4].

It does appear, based on the above calculations for $A_{2}$, that these identities cannot be used to construct new classes of elliptic-type Dunkl operators (thought the basic identity (2) does play a role in elliptic KZ-theory [9]). This is, however, scope for further investigation of this problem.

\section{Conclusions}

One recurrent theme in the theory of integrable systems is the tower of generalizations rational $\longrightarrow$ trigonometric $\longrightarrow$ elliptic,

and we have seen in this paper how the Frobenius-Stickelberger relation, via its $\vartheta$-function form, reflects this. The WDVV-equation are themselves an example of an integrable system; they arise as the zero-curvature conditions of the connection [6]

$$
\tilde{\nabla}_{X} Y=\nabla_{X} Y+\lambda X \circ Y
$$

and the solutions described in Theorem (2) (at least for the Weyl groups $A_{N}$ and $B_{N}$, and conjecturally for all Weyl groups) sit at the right of the following tower of generalizations:

$$
\left.\left\{\begin{array}{c}
\mathbb{C}^{N} / W \\
\text { oxeter group } \\
\text { orbit space }
\end{array}\right\} \quad\left\{\begin{array}{c}
\text { Extended affine Weyl } \\
\text { orbit space }
\end{array}\right\} \quad \longrightarrow \quad \begin{array}{c}
\mathbb{C}^{N+1} / \widetilde{W}(\mathfrak{g}) \\
\text { Jacobi group } \\
\text { orbit space }
\end{array}\right\}
$$

(technically the solutions are the corresponding 'almost dual' solutions [7] to the Frobenius manifold structures on these spaces and the solutions described in Theorem (2) correspond to the Frobenius manifolds constructed in $[2,18]$ ). These Jacobi group orbit spaces use in their construction properties of Jacobi forms $[8,21]$, functions which may be thought of as elliptic generalizations of $W$-invariant polynomials.

This tower of generalizations clearly need not stop at elliptic solutions. The $A_{N}$-Frobenius manifolds for the rational (indeed polynomial), trigonometric and elliptic cases have a Hurwitz space description in terms of the moduli space $H_{g, N}\left(k_{1}, \ldots, k_{l}\right)$ of branched coverings of the Riemann sphere. An interesting question is whether or not there is an orbit space construction for these more general spaces:

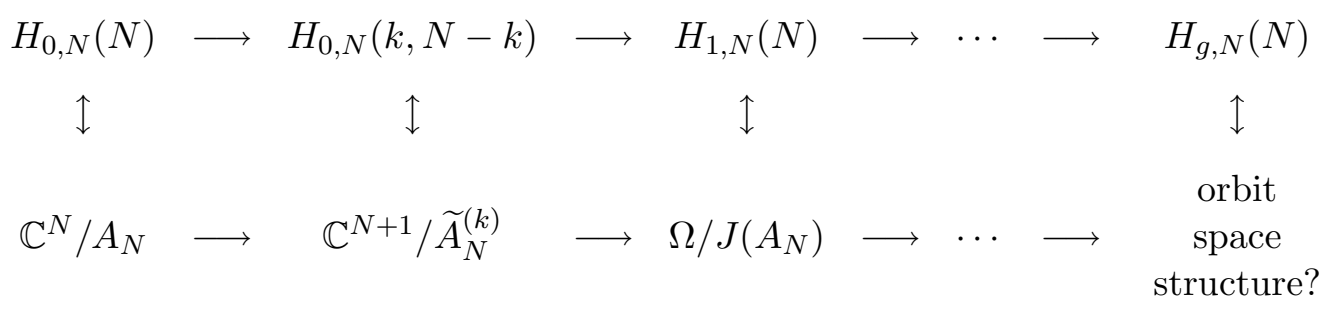

It seems sensible to conjecture that such an orbit space exists. One would expect Seigel modular forms to play a role instead of the modular forms used here. Higher genus Jacobi forms certain have been studied, but their use has yet to percolate into the theory of integrable systems. The development, and applications of, the neo-classical $\vartheta$-function identities studied in Section 4 remains to be done systematically. Higher genus analogues of these identities certain exist, since there exists almost-dual prepotentials on these Hurwitz spaces which, by construction, satisfy the WDVV equations. In the genus 0 and genus 1 cases, the prepotential is very closely related 
to the prime form on the Riemann surface. This may be the starting point for the development of a functional ansatz for the higher genus cases. Central to the results presented here are the quasi-periodicity and modularity properties of the elliptic polylogarithm, and these were obtained from the analytic properties of this function; the only role the analytic properties play were in the development of these transformation properties. It would be attractive if one could obtain these directly from the geometric properties of the prime form. This approach could then be used in the higher genus case where the analytic properties are likely to be considerably more complicated.

\section{Appendix A}

There are, unfortunately, many different definitions and normalizations for elliptic, numbertheoretic and other special functions. Here we list the definitions used in this paper. Let $q=e^{2 \pi i \tau}$, where $\tau \in \mathbb{H}$.

- $\vartheta_{1}$-function:

$$
\vartheta_{1}(z \mid \tau)=-i\left(e^{\pi i z}-e^{-\pi i z}\right) q^{\frac{1}{8}} \prod_{n=1}^{\infty}\left(1-q^{n}\right)\left(1-q^{n} e^{2 \pi i z}\right)\left(1-q^{n} e^{-2 \pi i z}\right) .
$$

The fundamental lattice is generated by $z \mapsto z+1, z \mapsto z+\tau$ and the function satisfies the complex heat equation $\vartheta_{1}^{\prime \prime}=4 \pi i \vartheta_{1, \tau}$.

- Bernoulli numbers:

$$
\frac{x}{e^{x}-1}=\sum_{n=0}^{\infty} B_{k} \frac{x^{n}}{n !}
$$

- Eisenstein series:

$$
E_{k}(\tau)=1-\frac{2 k}{B_{k}} \sum_{n=1}^{\infty} \sigma_{k-1}(n) q^{n}, \quad k \in 2 \mathbb{N},
$$

where $\sigma_{k}(n)=\sum_{d \mid n} d^{k}$.

- Dedekind $\eta$-function:

$$
\eta(\tau)=q^{\frac{1}{24}} \prod_{n=1}^{\infty}\left(1-q^{n}\right)
$$

- Polylogarithm function:

$$
\operatorname{Li}_{N}(z)=\sum_{n=1}^{\infty} \frac{z^{n}}{n^{N}}, \quad|z|<1
$$

Note that $\vartheta_{1}, E_{2}$ and $\eta$ are related:

$$
\frac{\eta^{\prime}(\tau)}{\eta(\tau)}=\frac{2 \pi i}{24} E_{2}(\tau)=\frac{1}{12 \pi i} \frac{\vartheta_{1}^{\prime \prime \prime}(0, \tau)}{\vartheta_{1}^{\prime}(0, \tau)} .
$$

These have the following properties under inversion of the independent variable:

$$
\tau^{-n} E_{n}\left(-\frac{1}{\tau}\right)=E_{n}(\tau), \quad n \geq 4
$$




$$
\begin{aligned}
& \tau^{-2} E_{2}\left(-\frac{1}{\tau}\right)=E_{2}(\tau)+\frac{12}{2 \pi i \tau} \\
& \eta\left(-\frac{1}{\tau}\right)=\sqrt{\frac{\tau}{i}} \eta(\tau),
\end{aligned}
$$

where in the last formula the square-root is taken to have non-negative real part. The polylogarithm has the inversion property:

$$
(-1)^{N-1} \operatorname{Li}_{N}\left(z^{-1}\right)=\operatorname{Li}_{N}(z)+\sum_{j=0}^{N} \frac{B_{j}(2 \pi \sqrt{-1})^{j}}{(N-j) ! j !}(\log z)^{N-j} .
$$

This may be used to analytically continue the function outside the unit disc to a multi-valued holomorphic function on $\mathbb{C} \backslash\{0,1\}$. For a discussion of the monodromy of the polylogarithm function see [20].

\section{Appendix B}

Here we present the identities corresponding to the $B_{2}$-root system. There are more than one identity in each of these two sets: this stems from the facts that the root systems have roots of different lengths.

Set $\mathbf{B}_{2}(\mathbf{a})$ :

$$
\begin{aligned}
& \left\{\begin{array}{c}
f^{(3,0)}(x)\left[f^{(2,1)}(y)-f^{(2,1)}(x+y)\right] \\
+f^{(3,0)}(x+y)\left[f^{(2,1)}(x)-f^{(2,1)}(x+2 y)\right] \\
+f^{(3,0)}(x+2 y)\left[f^{(2,1)}(x+y)-f^{(2,1)}(y)\right]
\end{array}\right\} \\
& +\left\{\frac{1}{2} f^{(1,2)}(x)-\frac{1}{2} f^{(1,2)}(x+2 y)-2 f^{(1,2)}(y)\right\}=0, \\
& \left\{\begin{array}{c}
f^{(3,0)}(y)\left[f^{(2,1)}(x+2 y)-f^{(2,1)}(x)\right] \\
+f^{(3,0)}(x+y)\left[f^{(2,1)}(x)-f^{(2,1)}(x+2 y)\right] \\
+2 f^{(3,0)}(x+2 y)\left[f^{(2,1)}(x+y)-f^{(2,1)}(y)\right]
\end{array}\right\} \\
& +\left\{f^{(1,2)}(x)+2 f^{(1,2)}(x+y)-2 f^{(1,2)}(y)\right\}=0 .
\end{aligned}
$$

\section{Set $B_{2}(b)$ :}

$$
\begin{aligned}
& \left\{\begin{array}{c}
f^{(3,0)}(x)\left[f^{(1,2)}(x+y)-f^{(1,2)}(y)\right] \\
+ \\
+f^{(3,0)}(x+y)\left[f^{(1,2)}(x)+f^{(1,2)}(x+2 y)\right] \\
+
\end{array}\right\}-2\left\{\begin{array}{c}
f^{(2,1)}(x+y) f^{(2,1)}(x) \\
+f^{(2,1)}(x+y) f^{(2,1)}(x+2 y)
\end{array}\right\} \\
& +\frac{1}{3}\left\{\begin{array}{c}
f^{(0,3)}(x)+2 f^{(0,3)}(x+y) \\
+f^{(0,3)}(x+2 y)+6 f^{(0,3)}(y)
\end{array}\right\}+\frac{1}{36} E_{4}(q)=0, \\
& \left\{\begin{array}{c}
f^{(3,0)}(y)\left[f^{(1,2)}(x+2 y)-f^{(1,2)}(x)\right] \\
+ \\
+4 f^{(3,0)}(x+2 y)\left[f^{(1,2)}(y)+f^{(1,2)}(x+y)\right] \\
+
\end{array}\right\}-4\left\{\begin{array}{c}
f^{(2,1)}(x+2 y) f^{(2,1)}(x+y) \\
+f^{(2,1)}(x+2 y) f^{(2,1)}(y)
\end{array}\right\} \\
& +\frac{1}{3}\left\{\begin{array}{c}
f^{(0,3)}(x)+8 f^{(0,3)}(x+y) \\
+f^{(0,3)}(x+2 y)+8 f^{(0,3)}(y)
\end{array}\right\}+\frac{1}{18} E_{4}(\tau)=0 .
\end{aligned}
$$




\section{Acknowledgments}

I would like to thank Harry Braden, who first showed me that (2) was just the FrobeniusStickelberger identity (1), and Misha Feigin and Andrew Riley for their comments and remarks.

\section{References}

[1] Beilinson A., Levin A., The elliptic polylogarithm, in Motives (Seattle, WA, 1991), Editors U. Jannsen, S. Kleiman and J.-P. Serre, Proc. Sympos. Pure Math., Vol. 55, Amer. Math. Soc., Providence, RI, 1994, Part 2, 123-190.

[2] Bertola M., Frobenius manifold structure on orbit space of Jacobi groups. I, Differential Geom. Appl. 13 (2000), 19-41.

Bertola M., Frobenius manifold structure on orbit space of Jacobi groups. II, Differential Geom. Appl. 13 (2000), 213-223.

[3] Chalykh O.A., Veselov A.P., Locus configurations and V-systems, Phys. Lett. A 285 (2001), 339-349, math-ph/0105003.

[4] Cherednik I., A unification of Knizhnik-Zamolodchikov and Dunkl operators via affine Hecke algebras, Invent. Math. 66 (1991), 411-431.

[5] Dunkl C., Differential-difference operators associated to reflection groups, Trans. Amer. Math. Soc. 311 (1989), 167-183.

[6] Dubrovin B., Geometry of 2D topological field theories, in Integrable Systems and Quantum Groups (Montecatini Terme, 1993), Editors M. Francaviglia and S. Greco, Lecture Notes in Math., Vol. 1620, Springer, Berlin, 1996, 120-348, hep-th/9407018.

[7] Dubrovin B., On almost duality for Frobenius manifolds, in Geometry, Topology, and Mathematical Physics, Amer. Math. Soc. Transl. Ser. 2, Vol. 212, Amer. Math. Soc., Providence, RI, 2004, 75-132, math.DG/0307374.

[8] Eichler M., Zagier D., The theory of Jacobi forms, Progress in Mathematics, Vol. 55, Birkhäuser Boston, Inc., Boston, MA, 1985.

[9] Calaque D., Enriques B., Etingof P., Universal KZB equations I: the elliptic case, math.QA/0702670.

[10] Feigin M., Trigonometric solutions of WDVV equations and generalized Calogero-Moser-Sutherland systems, arXiv:0802.0532.

[11] Feigin M., Veselov A.P., On the geometry of V-systems, in Geometry Topology and Mathematical Physics, Amer. Math. Soc. Transl. Ser. 2, Vol. 224, Amer. Math. Soc., Providence, RI, 2008, 111-123, arXiv:0710.5729.

[12] Frobenius G., Stickelberger L., Über die Addition und Multiplication der elliptischen Functionen, J. Reine Angew. Math. 88 (1880), 146-184.

[13] Heckman G.J., An elementary approach to the hypergeometric shift operators of Opdam, Invent. Math. 103 (1991), 341-350.

[14] Levin A., Elliptic polylogarithms: an analytic theory, Compositio Math. 106 (1997), 267-282.

[15] Marshakov A., Mironov A., Morozov A., A WDVV-like equation in $N=2$ SUSY Yang-Mills theory, Phys. Lett. B 389 (1996), 43-52, hep-th/9607109.

[16] Martini R., Hoevenaars L.K., Trigonometric solutions of the WDVV equations from root systems, Lett. Math. Phys. 65 (2003), 15-18, math-ph/0302059.

[17] Riley A., Strachan I.A.B., Duality for Jacobi group orbit spaces and elliptic solutions of the WDVV equations, Lett. Math. Phys. 77 (2006), 221-234, math-ph/0511048.

[18] Satake I., Frobenius manifolds for elliptic root systems, math.AG/0611553.

[19] Strachan I.A.B., Weyl groups and elliptic solutions of the WDVV equations, arXiv:0802.0388.

[20] Ramakrishnan D., On the monodromy of higher logarithms, Proc. Amer. Math. Soc. 85 (1982), 596-599.

[21] Wirthmüller K., Root systems and Jacobi forms, Compositio Math. 82 (1992), 293-354.

[22] Whittaker E.T., Watson G.N., A course of modern analysis. An introduction to the general theory of infinite processes and of analytic functions; with an account of the principal transcendental functions, reprint of 4 th ed. (1927), Cambridge University Press, Cambridge, 1996.

[23] Zagier D., The Bloch-Wigner-Ramakrishnan polylogarithm function, Math. Ann. 286 (1990), 613-624. 